\title{
Oral Diseases affect some 3.9 Billion people
}

\author{
Abstracted from \\ Marcenes W, Kassebaum NJ, Bernabé E, Flaxman A, Naghavi M, Lopez A, Murray CJ. \\ Global Burden of Oral Conditions in 1990-2010: A Systematic Analysis. \\ J Dent Res 2013; May 29. [Epub ahead of print] PubMed PMID: 23720570 \\ Address for correspondence: W. Marcenes, Institute of Dentistry, \\ Barts and The London School of Medicine and Dentistry, Queen Mary University of London, \\ London, UK. E-mail: w. marcenes@qmul.ac.uk
}

\section{Question: What is the global burden of untreated caries, severe periodontitis and severe tooth loss in 2010 ?}

Data sources Medline, Embase, Lilacs.

Study selection Published and unpublished observational populationbased studies presenting information on the prevalence, incidence, case fatality and cause-specific mortality related to untreated caries, severe periodontitis and severe tooth loss between January 1980 and December 2010. There were no language restrictions. Study quality was assessed using the STROBE checklist (http://www.strobe-statement.org/). Data extraction and synthesis Prevalence estimates were calculated on the database for all age-gender-country-year groups using a specifically developed Bayesian meta-regression tool. Disabilityadjusted life-years (DALYs) and years lived with disability (YLDs) metrics were used to quantify the disease burden. Disability weights were calculated based on population-based surveys in five countries (USA, Peru, Tanzania, Bangladesh and Indonesia) and an open Internet survey. Uncertainties in estimates were examined using Monte Carlo simulation techniques with uncertainty levels presented as the 2.5 th and 97.5 th centiles, which can be interpreted as a $95 \%$ UI.

Results Oral diseases remain highly prevalent in 2010 affecting 3.9 billion people. Untreated caries in permanent teeth was the most prevalent condition evaluated for the entire GBD (Global Burden of Disease) 2010 Study with a global prevalence of $35 \%$ for all ages combined. Severe periodontitis and untreated caries in deciduous teeth were the 6 th and 10th most prevalent conditions, affecting, respectively, $11 \%$ and $9 \%$ of the global population. Oral conditions combined accounted for 15 million DALYs globally ( $1.9 \%$ of all YLDs and $0.6 \%$ of all DALYs), implying an average health loss of 224 years per 100,000 people. DALYs due to oral conditions increased $20.8 \%$ between 1990 and 2010, mainly due to population growth and aging. While DALYs due to severe periodontitis and untreated caries increased, those due to severe tooth loss decreased. Conclusions The findings highlight the challenge in responding to the diversity of urgent oral health needs world-wide, particularly in developing communities.

\section{Commentary}

This review presents the oral health findings of the Global Burden of Disease Study(GBD) 2010 study which was funded by the Bill \& Melinda Gates Foundation and undertook a systematic comparison to provide estimates of the burden of 291 diseases worldwide. ${ }^{1}$
A sound methodological approach has been taken to gather the information for this analysis, but as the authors note in their discussion, there are inherent challenges owing to the variation in the reporting of oral disease and incomplete data.

In addition some studies had to be excluded as one of the models used relied on fixed effects being relatively constant with respect to time and age, and this poses problems for both dental caries and periodontal disease. As a result the DALYs calculation was only based on the prevalence of total tooth loss.

The GBD case definitions focused only on the most severe oral conditions, and as the authors point out, some disability is caused by restored teeth, mild to moderate tooth loss and periodontitis. As oral conditions have effects on the ability to eat and a psychosocial impact, more information on these elements would lead to a better understanding of the burden of these health states. While it is encouraging that DALYs due to severe tooth loss decreased by $12.3 \%$, those due to severe periodontitis and untreated caries in deciduous and permanent teeth increased by $57.3 \%, 5.3 \%$ and $38.1 \%$ respectively. Some of these changes are associated with population growth and aging.

This review, which probably underestimates the impact of oral diseases, highlights the significant challenge that these largely preventable oral diseases still pose. In 2003 the WHO proposed some goals, objectives and targets for oral health that could be adopted for regional or locally relevant goals. One of these was to reduce social inequalities in oral health. However, If we are to develop sustainable approaches to improving oral health we will need to reorientate oral health research, practice and policy toward a 'social determinants' model as outlined by Sheiham et al.,, 3,4 as traditional models of oral health service delivery have failed to deliver significant improvements in oral health and had little or no impact on oral health inequalities. The International Association of Dental Research Global Oral Health Inequalities Research Agenda (IADRGOHIRA $($ ) is a welcome initiative and only time will tell if this will lead to significant improvements in oral health inequalities.

Derek Richards Centre for Evidence-based Dentistry, Oxford, UK.

1. Murray C), Ezzati M, Flaxman, AD et al. GBD 2010: design, definitions, and metrics. Lancet 2012; 380: 2063-2066.

2. Hobdell M, Petersen PE, Clarkson J, Johnson N. Global goals for oral health 2020. Int Dent J 2003; 53: 285-288.

3. Sheiham A, Alexander D, Cohen L, et al. Global oral health inequalities: task group implementation and delivery of oral health strategies. Adv Dent Res 2011; 23: 259-267.

4. Sheiham A. Major changes in strategies are needed to promote oral health worldwide. J Public Health Dent 2013; 73: 87-88.

Evidence-Based Dentistry (2013) 14, 35. doi:10.1038/sj.ebd.6400925 